\title{
A multi-objective optimization platform for artificial lighting system in commercial greenhouses
}

\author{
Ying Qu', Anders Clausen and Bo Nørregaard Jørgensen \\ From 1st Energy Informatics.Academy Conference Asia \\ Beijing, China . 29-30 May 2021
}

\author{
*Correspondence: yqu@mmmi.sdu. \\ dk \\ The Maersk Mc-Kinney Moller \\ Institute, University of Southern \\ Denmark, Campusvej 55, 5230 \\ Odense, Denmark
}

\begin{abstract}
Limited natural daylight in Nordic Countries means artificial lighting is a critical factor in industrial plant production. The electricity cost of artificial lights accounts for a large percentage of the overall cost of plant production. The optimal use of artificial lighting in plant production can be formulated as a multi-objective problem (MOP) to achieve optimal plant growth while minimizing electricity cost. In previous work, for solving this MOP, a Genetic Algorithm (GA) was used to create a Pareto Frontier (PF), which contains solutions representing a trade-off for using artificial lighting against plant production objectives. The PF was updated immediately once a nondominated child-solution was found by comparing the dominance with solutions in the PF. Besides, in addition to the PF, the initial random population is also reused as a parent source in the evolution process. When the genetic evolution process terminated, a priority-based selection mechanism was used to select a final solution from the PF. In this paper, an alternative evolution strategy is proposed and compared with the previous GA evolution strategy. By this alternative strategy, all child-solutions are only compared with their parents during the evolution process, and the non-dominated child-solutions are collected into a candidate list. The PF is then updated at the end of each generation by comparing solutions on the PF with the collected candidate solutions. In this alternative strategy, the PF is the only source of parent-solution during the evolution process. In addition, a posterior normalization is implemented in the dominance evaluation, and social welfare metrics (SWs) are applied as an alternative to the priority-based selection mechanism to avoid the explicit ranking of objectives. The experimental results show that the proposed alternative evolution strategy outperforms the previous strategy on dramatically avoiding local minima.
\end{abstract}

Keywords: Multi-objective optimization problem (MOP), Social Welfare metrics (SWs), Commercial greenhouse, Multi-objective evolutionary algorithm (MOEA), Genetic Algorithm (GA) 


\section{Introduction}

Greenhouses are complex systems that can provide the required environment for plant production. Greenhouses are high energy-consuming facilities with approximately $50 \%$ energy consumption cost of the whole production process (Shen et al. 2018). There are two types of energy consumption in a greenhouse system - the basic energy consumption and the actuator driving consumption. The basic energy consumption, including artificial lighting, dehumidification, heating, cooling, etc., accounts for more than $90 \%$ of the total energy consumption in the greenhouse (Kjaer et al. 2011; Hemming et al. 2017). Aside from this, artificial lighting systems consume approximately $75 \%$ of the total energy consumption (Sørensen et al. 2016a; Kjaer et al. 2012). This makes the energy-efficient operation of artificial light critical in commercial plant production.

Several approaches have defined this as a multi-objective optimization problem (MOP). In (Aaslyng et al. 2003), a greenhouse climate control system, "IntelliGrow," was suggested to reduce energy consumption by optimizing the use of heating and artificial lighting according to natural irradiance while maintaining the performance in plant production. The authors committed experiments of IntelliGrow on two greenhouses in Denmark for 6 months and compared the results of the experiments with historical data from plant production at the site. Comparing energy consumption during the use of IntelliGrow with the energy use recorded in historical data suggested that IntelliGrow was able to reduce energy consumption drastically. However, when IntelliGrow calculates the optimal climate control parameters, such as $\mathrm{CO} 2$ concentration and temperature, only the irradiance that is obtained from natural light is considered. Thus, the contribution from artificial light is not considered in the optimization. Further, the optimization in IntelliGrow does not consider the physical properties of the installed hardware, such as the effectiveness of the ventilation system and the heating system, which can also affect the optimization in a real scenario.

In (Markvart et al. 2008), a Genetic Algorithm (GA) is proposed to solve the problem. Their application, DynaLight, models each growth compartment as an optimization context where the optimization goal is to find an optimal schedule for using artificial light, considering multiple objectives - including objectives that seek to minimize electricity cost, ensure sufficient artificial lighting for the plants while considering natural irradiation forecasts and taking into account grower-specific preferences towards the light schedule. The schedule for the use of artificial lighting is referred to as the light plan. This approach creates a PF of Pareto optimal light plans (Hashem et al. 2017). In DynaLight, the PF is updated once a child solution is created through mutation or crossover by comparing the dominance of this new solution with the current PF. For comparing the dominance, two comparators are involved: first against the objectives with the highest priority and then against all of the domain objectives. As a result, only the solutions that are nondominated against the highest priority objectives can remain in the PF. In the GA evolution process, parent solutions are randomly selected from both the initial random population and the current PF. Once an optimization epoch terminates, a single solution is selected from the final Pareto set. This is done using a ranked selection mechanism that considers the configured importance of the objectives. However, due to the nature of the selection mechanism, which considers the unnormalized evaluation value from each objective towards each solution, the ranking has to consider the range of returned, absolute values from each objective. 
To date, no analysis has been performed of the consequences of this approach concerning the coverage of the PF or the consequences of the selection mechanism. To this end, this paper introduces an alternative version of DynaLight, DynaLight IND. In DynaLight IND, all the child solutions created by mutation and crossover and nondominated children compared with their parent(s) are collected into a candidate list during evolution. Then, the PF is updated by comparing the dominance of the solutions in the candidate list with the current PF after all the evolutions of a generation, rather than updating PF once a child solution is created. Differentiating with DynaLight, only one comparator, against all the domain objectives, is utilized in DynaLight IND. Through these modifications, DynaLight IND can enhance the coverage of PF. Besides, DynaLight IND accelerates the computational speed by reducing the computational cost by only involving the current PF as the parent source. The purpose is to analyze the impact of the previous GA evolution strategy on the coverage of the PF. Further, an alternative selection mechanism that uses posteriori optimization normalization (Umair et al. 2019) is employed in DynaLight IND to enable the ranking of objectives independent of the nature of their evaluation function.

Simulations using equal environment parameters across DynaLight and DynaLight IND have been conducted to compare the found Pareto sets and the final selected solution between the two implementations. The results of the experiments show that DynaLight IND can enhance the PF coverage up to 600 times compared to that of DynaLight. Further, the use of the alternative selection mechanism enables the selection of a final solution from the PF set independent of objective evaluation function nature with comparable qualities to that of the original rank order selection mechanism while adhering to the Separation of Concerns principle.

The remaining part of this paper is organized as follows. In section "Problem description," the multi-objective problems (MOPs) concerned in this paper is introduced. Section "Approach" introduces the changes made to DynaLight, including changes made to the evolution implementation of GA and the use of a posteriori normalization combined with Social Welfare metrics (SWs) (Chevaleyre et al. 2006) to enable selection from the Pareto set without considering the nature of the objective functions. Section "Experimental configuration" introduces the experiments conducted with the two versions of DynaLight, and section "Results" presents the results of those experiments. The experimental results are analyzed and compared in section "Discussion," and the conclusion of this paper is stated in section "Conclusions."

\section{Problem description}

In this section, the architecture of the lighting system in commercial greenhouses is introduced, and the multi-objective problem associated with its operation is explained.

\section{Artificial lighting system of greenhouses}

Figure 1 illustrates the concepts of the lighting system in a commercial greenhouse. The lighting system is comprised of a data pool component, the optimization application (here, DynaLight or DynaLight IND), hardware facilities, and the hardware controller component, which also contains a climate computer, aside from the controllers, that is used to send 


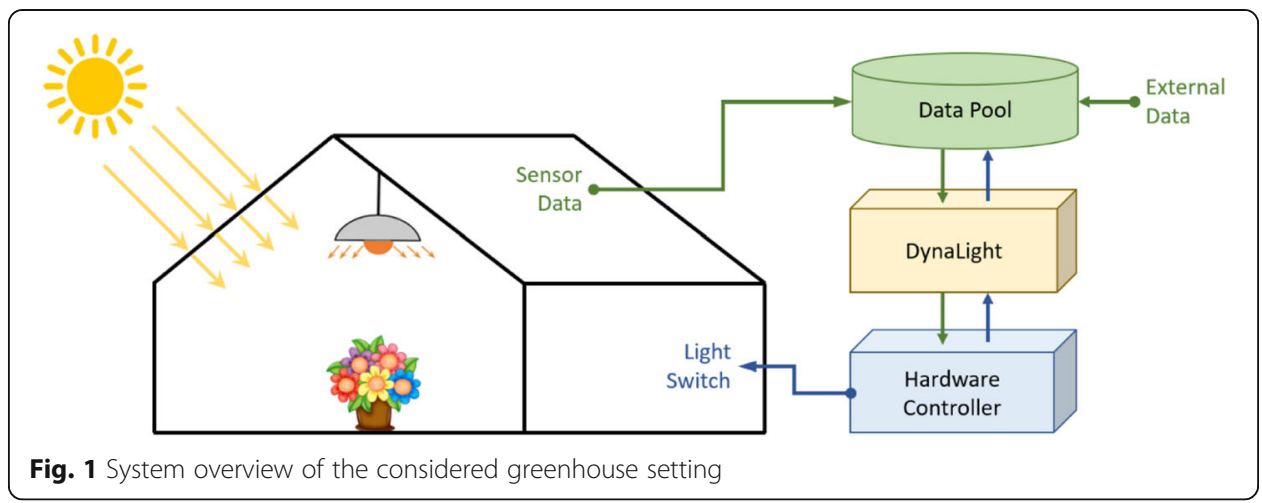

control parameters to the hardware controllers and for monitoring purposes (Albadi and El-Saadany 2008; Ma et al. 2020; Warde 2015).

The data pool collects data relevant to the greenhouse system. These data constitute data from the local sensors, including indoor- and outdoor temperatures, the internal humidity and local photosynthetically active radiation (PAR) values as well as relevant external data such as weather forecasts and electricity prices. DynaLight uses this information as an input used in optimization to an optimal plan for using artificial light, which ensures cost-effective use of electricity while maintaining the quality of the plant production.

The output of DynaLight is a light plan containing actuation points for the artificial lights. The light plan is represented by a switching schedule; that is, a sequence of ON and OFF status signals. This sequence of switch signals for the artificial lights is sent to the climate computer. The climate computer converts the switch status to a drive signal to control the lamps in the greenhouse (Anthony Howard et al. 2020).

\section{Objective definition}

In DynaLight and DynaLight IND, objectives are expressed as evaluation functions that evaluate a suggestion for a light plan by returning a cost. The target of individual objectives is to minimize the cost of the corresponding evaluation function. The MOP concerned by DynaLight includes seven independent objectives (Sørensen et al. 2016a; Rytter et al. 2012). The form of the MOO for this problem is shown in Formula (1).

$$
\min f_{m}(\boldsymbol{X}), m \in[1, M]
$$

s. $t . X=\left[x_{1} \cdots x_{n} \cdots x_{N}\right], x_{n}=\{0,1\}, n \in[1, N]$, where, $f_{m}(X)$ denotes the $m^{\text {th }}$ objective. $M=7$, which means there are seven objectives are involved in this MOP. $X$ corresponds to a light-plan and $x_{n}$ is the artificial light status of the $n^{\text {th }}$ hour and $N \in[1,72]$ is the size of vector $X$. As the light-plan is scheduled for every $72 \mathrm{~h}, N$ represents the number of remaining hours in a $72 \mathrm{~h}$ schedule period. The details of the individual objectives are explained below.

\section{"PAR sum balance" objective}

The "PAR sum balance" objective aims to calculate the PAR balance between the PAR sum based on a light plan and the PAR goal in a period. The balance is calculated over a five-day-period defined by current day, two past days and two future days. "Current day" here represents the exact date that the simulation starts. Generally, there are two 
sources of PAR in greenhouses: Natural irradiation and artificial light. The associated objective function is defined as:

$$
\begin{aligned}
& f_{1}(\boldsymbol{X})=\left(P_{\text {hist }}+P_{\text {exp }}+P_{\text {art }}\right)-P_{\text {goal }} \\
& P_{\text {hist }}=\sum_{i=1}^{n-1} p_{i} \\
& P_{\text {exp }}=\sum_{j=n}^{N} p_{j} \\
& P_{\text {art }}=P_{\text {SON-T }} N_{\text {light }} \sum_{n}^{N} x_{n}
\end{aligned}
$$

where, $f_{1}(\mathrm{X})$ represents the PAR balance between the PAR sum and the goal in the five-day-period. The 5 days cover the historical 2 days, the current day and the future 2 days. $P_{\text {hist }}$ is the historically achieved PAR in the greenhouse. $P_{\text {hist }}$ is the PAR sum of each hour from all sources in the past period in the greenhouse compartment. In a real situation, $P_{\text {hist }}$ is collected by the local sensors. The historical period involves the past 2 days and the past hours in the current day. $P_{\exp }$ is the sum of the expected PAR in the future. This includes the future 2 days and the remaining hours in the current day. $P_{\exp }$ is the indoor PAR that is converted from the outdoor natural PAR, which is collected from the weather forecast provider Conwx. $P_{a r t}$ is the PAR that is provided by the artificial lights in the future hours in the light-plan period, which covers $(N-n+1)$ hours. The converted PAR from artificial lights is impacted by the light type and the number of lights. In this problem, $P_{S O N-T}$ represents the PAR amount that a SON-T light provides, which is the type the artificial lights in this problem; $N_{\text {light }}$ is the number of lights that are installed in the greenhouse compartment. $P_{\text {goal }}$ represents the five-day goal of PAR, which is described in Formula (6).

$$
P_{\text {goal }}=N_{\text {period }} \times P_{\text {day }}
$$

where $N_{\text {period }}=5$ and $P_{\text {day }}$ is the PAR goal per day.

\section{"Sufficient PAR sum" objective}

The aim of the objective "Sufficient PAR sum" is to determine whether the PAR sum of a light plan solution can fulfill the PAR goal in a period or not. The evaluation results of this objective are Boolean values, which are defined in Formula (7). If the PAR balance is non-negative, it means the according solution can satisfy the period goal.

$$
f_{2}(\boldsymbol{X})=\left\{\begin{array}{c}
0, f_{1}(\boldsymbol{X}) \geq 0 \\
1, f_{1}(\boldsymbol{X})<0
\end{array}\right.
$$

The calculation of $f_{1}(X)$ is presented in Formula (2). The difference is that for this objective, $N_{\text {period }}=3$ in the calculation of $P_{\text {goal }}$ in Formula (6). Thus, the "Sufficient PAR sum" objective focuses on the PAR goal for the current day as well as two days into the future. 


\section{"Fixed Light Hours" objective}

The "Fixed Light Hours" objective allows domain experts to influence the outcome of the optimization directly. This objective aims to determine whether a solution fulfills the status requirement of artificial lights at the fixed light hours as configured by a grower. The cost value of this objective $f_{3}(X) \in R$ is calculated by the light status of an individual hour in the light-plan $X$ XOR the fixed light schedule $X_{f i x}$, which is denoted in Formula (8).

$$
f_{3}(\boldsymbol{X})=\boldsymbol{X} \oplus \boldsymbol{X}_{f i x}
$$

Therefore, if the light plan does not equal the corresponding schedule requirements at specific hours, $f_{3}(X)$ is non-zero. The light-plan must fulfil the requirement of fixed hours, so only the solutions that have zero evaluation cost of this objective are acceptable choices from the final Pareto set.

\section{"Light interval" objective}

Many greenhouse compartments use SON-T lights for providing artificial light to the plants in the compartment. SON-T lights cannot tolerate frequent changes of switch status due to the physical properties of the light. In this case, the smallest frequency of change of the switch status is $5 \mathrm{~min}$. "Light Interval" objective aims to make sure that the lights are not toggled more frequently.

The evaluation function is in Formula (9).

$$
f_{4}(\boldsymbol{X})=x_{1} \oplus x_{\text {curr }}
$$

The evaluation result of $f_{4}(X)$ is a Boolean value. When the simulation interval is set to shorter than $5 \mathrm{~min}$, in the current one-hour-slot, the evaluation result $f_{4}(\boldsymbol{X})$ is zero if $x_{1}$ in $X$ is the same as the current light status $x_{\text {curr }} . x_{1}$ indicates the light status for the current hour in the light plan. Otherwise, the evaluation result returns one. Only the light-plan solutions with the same switch status with the current light status for the current one-hour slot are considered acceptable selections from the final Pareto set.

\section{"Minimum Light Switches" objective}

This objective aims to prolong the lifetime of the electrical equipment, such as the light bulbs, the switch system, etc., by minimizing the switching frequency. The cost of this objective $f_{5}(\boldsymbol{X})$ is the number of the light status changes in a light-plan solution, which is represented in Formula (10).

$$
f_{5}(\boldsymbol{X})=\sum_{i=1}^{N-1} x_{i} \oplus x_{i+1}
$$

\section{"Cheap light" objective}

The "Cheap Light" objective aims to minimize the electricity cost of a light plan. The Nordic Countries allow access to a spot market with hourly variation in electricity prices for large electricity consumers. In such a context, the electricity cost of switching on light varies from hour to hour. The aim of this objective is to select the cheaper solutions. The evaluation function is provided in Formula (11). 


$$
f_{6}(\boldsymbol{X})=P_{S O N-T} N_{\text {light }} \cdot \boldsymbol{X} \cdot \boldsymbol{P}_{e l}
$$

As in Formula (5), $P_{S O N-T}$ and $N_{\text {light }}$ represent the PAR amount that a SON-T light provides and the number of installed lights, respectively. $\boldsymbol{P}_{e l}=\left[p_{1} \cdots p_{i} \cdots p_{N}\right]^{T}, i \in[1, N]$, where $p_{i}$ represents the electricity price of $i^{\text {th }}$ hours.

\section{"Minimum Artificial Light" objective}

The aim of this objective is to minimize the excessive use of artificial light when the natural light is intense. This comes from the fact that the contribution from artificial light is significantly lower when outside irradiation is high. As shown in Formula (12), $N_{\text {larger }}$ denotes the number of hours in a light-plan solution when the indoor light that is converted from the natural light is larger than a given level, which is $100 \mu \mathrm{mol} / \mathrm{m}^{2} / \mathrm{s}$ in this case. The lower of $f_{7}(X)$, the more efficient the use of artificial light.

$$
f_{7}(\boldsymbol{X})=N_{\text {larger }}
$$

\section{Approach}

The overall flow in the optimization process is identical across DynaLight and DynaLight IND. An overview of this process is shown in Fig. 2. The key differences between DynaLight and DynaLight IND are summarized in Table 1. Changes made to DynaLight IND, including changes to the GA implementation, the posterior normalization, Relative Importance Graph (RIG), and SWs, are elaborated in this section.

Figure 2 shows that inputs and implementation configurations are collected from the database and the Internet when the optimization is started. Inputs are comprised of the

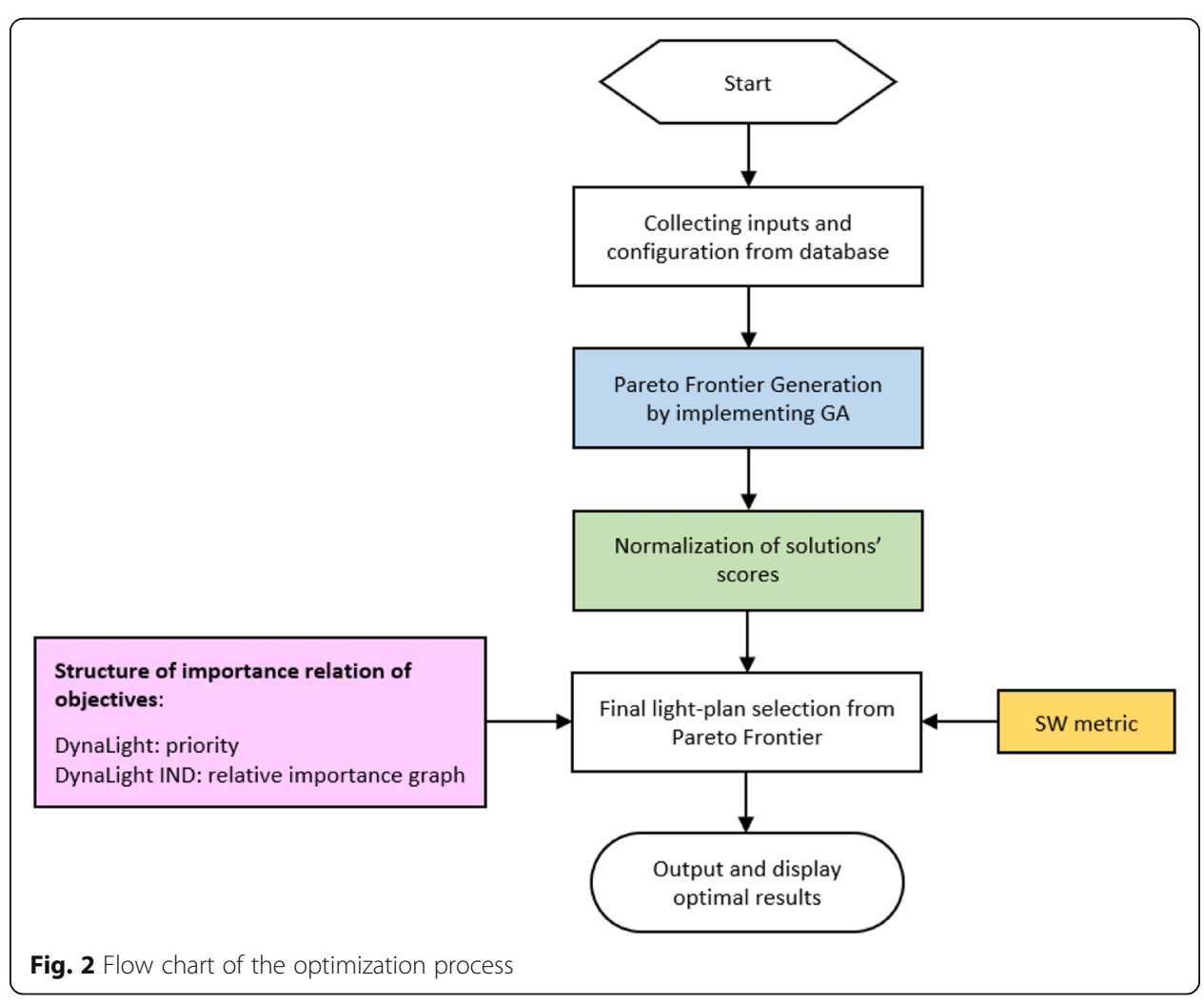


Table 1 The comparison of the two versions

\begin{tabular}{|c|c|c|}
\hline Aspect & DynaLight & DynaLight IND \\
\hline $\begin{array}{l}\text { GA } \\
\text { implementation } \\
\text { strategy }\end{array}$ & $\begin{array}{l}\text { - Updating PF once a new child-solution } \\
\text { is created. } \\
\text { - Two comparators: first against highest } \\
\text { priority objectives and then against all } \\
\text { domain objectives. } \\
\text { - The initial random population is another } \\
\text { parent-solution source aside from the } \\
\text { current PF. }\end{array}$ & $\begin{array}{l}\text { - Collecting all non-dominated child-solutions, } \\
\text { compared with parents, into a candidate list, } \\
\text { then comparing all candidates with current PF } \\
\text { at the end of each generation. } \\
\text { - One comparator: against all domain } \\
\text { objectives. } \\
\text { - The current PF is the only parent-solution } \\
\text { source. }\end{array}$ \\
\hline $\begin{array}{l}\text { Evaluation } \\
\text { strategy }\end{array}$ & No normalization & Posteriori normalization \\
\hline $\begin{array}{l}\text { Objective } \\
\text { relation }\end{array}$ & priority & Relative Importance Graph \\
\hline sw & Only one SW metric & $\begin{array}{l}\text { Multiple SW metrics (Adler 2012; Allan and } \\
\text { Feldman 2006; Chang 2000; Varian 2010) }\end{array}$ \\
\hline
\end{tabular}

electricity price forecast, the weather forecast, hardware properties, and sensor data. Two types of configurations are supported by DynaLight and DynaLight IND: structure configurations and implementation configurations. Structure configurations determine which objectives to include in the optimization, the relationship between objectives (rank for DynaLight and the RIG configuration, along with SW metrics for DynaLight IND). The implementation configurations are comprised of the environmental requirements of a specific context, such as the goal of PAR for 1 day, the interval of optimization calculation, etc.

Once inputs are gathered and configuration is done, the optimization phase starts. For each generation of GA calculation, a set of non-dominated solutions are selected using Pareto optimality (Fonseca and Fleming 1998), which will participate in the next evolution until the termination conditions are achieved. For DynaLight and DynaLight IND termination criteria includes the number of generations or a time constraint whichever is met first.

After the optimization, the evaluation values provided by each objective to each solution in the Pareto Set are normalized in DynaLight IND. The utilized posterior normalization method in DynaLight IND is elaborated later in this section.

For selecting the final solution from the Pareto Set, DynaLight ranks solution based on their evaluation by the correspondingly ranked objectives. Specifically, solutions that are best for objectives with rank 0 form a subset, from which the solutions which are best for rank 1 objectives are selected and so on until only a single solution remains. For DynaLight IND, the RIG is traversed and each node in the RIG performs a filtering mechanism similar to that of DynaLight, except here social metrics are configurable at each node in the RIG.

\section{GA implementation strategy}

The evolutionary process of DynaLight and DynaLight IND is shown in Listing 1 and Listing 3, respectively. The implementation of function "AddNonDominatedSolutionToPF" in DynaLight and function "CheckDomin" in DynaLight IND are elaborated in Listing 2 and Listing 4, respectively. Regarding the strategies of evaluating whether a 
newly created child solution is non-dominated and can be added to the PF, there are multiple differences between DynaLight and DynaLight IND.

Firstly, in the function "AddNonDominatedSolutionToPF" of DynaLight, once a new child solution is evolved, the dominance of it needs to be compared with all solutions in the current PF (for loop Line 2-15 in Listing 2). However, in DynaLight IND, after a child solution is evolved by mutation or crossover, the dominance of it is compared only with its parent(s) (Line 7 and 14 in Listing 3).

Secondly, there are two comparators involved in the dominance evaluation in DynaLight. In the first comparator, the dominance comparison of solutions is performed against the objectives in the highest priority (Line 3 in Listing 2). Then, in the second comparator, if the new child solution is non-dominated by the current PF, it is compared against the objectives with lower priorities (Line 9 in Listing 2), respectively. Instead, in DynaLight IND, only the dominance comparator against all the domain objectives is involved (Line 2 in Listing 4).

Thirdly, according to the dominance comparison results, in DynaLight, the current PF is immediately updated once a non-dominated child solution is evolved through mutation or crossover (Line 6, 9, 14, 16, and 18 in Listing 1). Once a child solution is non-dominated and can be added to the PF, the dominated solutions in the PF are removed. Instead, in DynaLight IND, the evolved non-dominated children, compared with their parents, are added to a candidate list (Line 20 in Listing 3). Only when reaching the terminal of one generation will the non-dominated solutions in the candidate list be added to the PF and the dominated solutions are removed. Therefore, the $\mathrm{PF}$ is not updated until the end of the generation.

Finally, in DynaLight, initialRandomPop (Listing 1) represents the initial random population generated for the initial parent population at the beginning of the evolution process, so the solutions in it are without any dominance checking. Aside from the current PF, initialRandomPop also participates in the evolution process as a parent-solution source. Solutions are selected randomly from initialRandomPop as parent-solution(s) for mutation and crossover (Line 7, 15, and 17 in Listing 1). Instead, in DynaLight IND, the current $\mathrm{PF}$ is the only parent-solution source to participate in mutation and crossover (Line 5, 11 and 12 in Listing 3), and initialRandomPop is only utilized for generating the initial PF.

\section{A posteriori normalization}

To enable the comparison of the cost, with different ranges, among the objectives in this MOP, a posteriori normalization has been applied in DynaLight IND (Umair et al. 2019). Here, the value spans of the objective functions for solutions in the Pareto set are considered to normalize the objective function values on a scale from 0 to 1 . This is different from a priori normalization in that no global knowledge on the search space is needed upfront and the dynamic properties of the objective functions are preserved since their span may differ between optimization epochs.

Assuming the sizes of the solution vector $S$ is $n$, which maps the PF that is generated by GA, and $s_{i} \in S$, where $i \in[0, n)$. There are $m$ elements in the objective vector is $C$, and $c_{j}$ represents one of the objectives, where $j \in[0, m)$. The posterori normalization can be described as Formula (13). 


$$
q_{c_{j}}^{\prime(i)}=\frac{q_{c_{j}}^{(i)}-q_{c_{j} \cdot \min }}{q_{c_{j} \cdot \max }-q_{c_{j^{\prime}} \cdot \min }}
$$

where, $q_{c_{j \cdot} \cdot \min }$ and $q_{c_{j \cdot} \cdot \max }$ are the actual maximum and the minimum cost of the PF against the objective $c_{j}$. $q_{c_{j}}^{(i)}$ is the cost of solution $s_{i}$ against objective $c_{j}$, and $q_{c_{j}}^{\prime(i)}$ is the posterori normalized cost of $q_{c_{j}}^{(i)}$.

\section{Relative importance graph (RIG)}

During selecting the optimal light plan from the PF, the selected solution needs to fulfill the objectives that are relatively most important to the overall goals of the system here, the production of plants. For example, reducing electricity consumption is more important than minimizing light switching times but less important than fulfilling the light requirement in fixed hours. This is because profit gained by favoring cheap electricity consumption is higher than the saving achieved through minimizing light switching, but ensuring the performance of the production is more important than saving money on electricity.

In DynaLight, the objective relations are denoted by the concept of priority. The priority is represented by a real number $P \in R$, and the larger $P$ is, the lower priority of a particular objective is. Hence, $P=0$ represents the highest priority. However, since the objective costs are not normalized for the SW calculation in DynaLight, objectives in the same priority must have the same cost scales in their objective functions to avoid a situation where one objective dominates another in the selection process.

In DynaLight IND, the importance relations among the objectives are described by the relative importance graph concept, which is introduced in $[14,27] . R_{a b}=\left\{r_{12}, \ldots\right.$, $\left.r_{a b}, \ldots, r_{(m-1) m}\right\}$ is the vector of the relations between every two objectives in the system, where $m$ is the number of objectives, and $r_{a b}$ represents the relation between $c_{a}$ and $c_{b}$, where $a \in[1, m), b \in(1, m]$. As shown in Table 2, there are three types of relations between two objectives, which are represented by $-1,0$ and 1 .

Once the relative importance relations between every objective are established, a relative importance graph can be generated accordingly (Umair et al. 2019; Clausen et al. 2020). A RIG is a directed graph, and there are two key elements in it, which can be described as $R I G=\langle V, E\rangle . V=\left\{V_{0}, \ldots, V_{i}, \ldots, V_{n}\right\}$ represents the group of vertexes in a RIG, where $i \in[0, n](n \in N)$, and there are $(n+1)$ vertexes in the particular RIG. $V_{0}$ means the Vertex Root. $C_{i}$ is the vector of objectives in $V_{i}$. There can be one or multiple objectives in $C_{i}$ even when the cost scales of objectives which are in the same node are inconsistent because the costs have been normalized as described in the section "A posteriori normalization." All objectives in a specific vertex have the same relative importance. $E=\left\{E_{01}, \ldots, E_{j k} \ldots, E_{(n-1) n}\right\}$ is a group of directed edges which connect vertexes, where $j \in[0, n-1]$ and $k \in[1, n]$, and there are $n$ edges in a particular RIG.

Table 2 Relations between objectives

\begin{tabular}{lll}
\hline$r_{a b}$ & Symbol & Definition \\
\hline-1 & $c_{a} \rightarrow c_{b}$ & Objective $a$ is relatively more important than objective $b$. \\
0 & $c_{a} \leftrightarrow c_{b}$ & Objective $a$ and objective $b$ are equally important. \\
1 & $c_{a} \leftarrow c_{b}$ & Objective $a$ is relatively less important than objective $b$. \\
\hline
\end{tabular}


The relative importance relationship between two vertexes is represented by an edge $E_{j k}$, which means the edge comes from $V_{j}$ and goes into $V_{k}$, and the objective(s) in $C_{j}$ are relatively more important than the objective(s) in $C_{k}$.

Figure 3 demonstrates the flow of selecting a final solution from a PF using the generated RIG. On the first vertex in the RIG, which is indexed as Vertex Root, the performance of all the solutions in the PF are evaluated according to the SW configured for that node. This generates a subset of solutions that is fed into the next vertex. This process continues until there is only one solution left in the subset or all the vertexes have been traversed.

During traversal of the RIG, one of the following three outcomes may be observed:

(1) If there is only one objective in a specific vertex, the SW selection does not influence the outcome. Instead, only the best solutions from the perspective of the objective can be added to the subset resulting from the traversal through this node.

(2) If there is only one solution in the solution set at any given point, this solution will automatically be the best for all objectives in the node. The optimization process

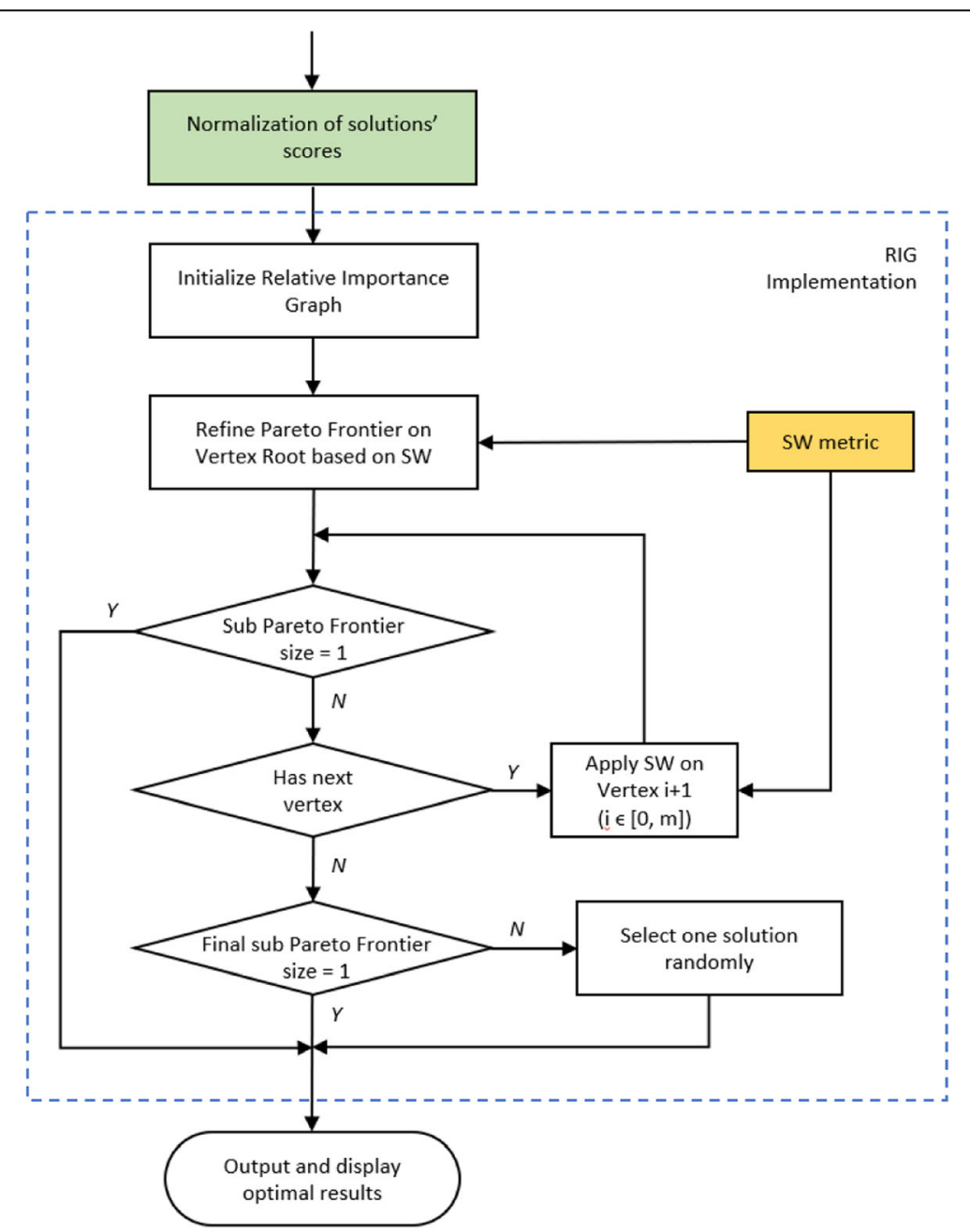

Fig. 3 Flow chart of the selection process of light-plan from PF with RIG 
terminates even if there are vertexes that have not been traversed, as no other outcome can be expected from any nodes below the reached node.

(3) If there is more than one solution left in the subset of the last vertex in the RIG, then the final solution will be chosen as a random solution in the set, as all solutions in the set are considered to be equally good from the perspective of the objectives in the optimization. Here equally good means the dominance evaluation of these solutions against the objectives in the current vertex are the same.

\section{Experimental configuration}

In this section, the experimental configurations for the simulations on the two versions of DynaLight are described, including the environmental condition, the simulation configuration and the objective importance relation setup.

\section{Environmental condition}

In a commercial greenhouse system, there are several compartments that provide environmental conditions for different growing stages of plants. The greenhouse model that is utilized in this simulation represents one short-day compartment of a horticultural greenhouse system. The key environmental parameters of the greenhouse compartment model related to lighting optimization are listed in Table 3. The type of artificial lights is SON-T. The physical feature of SON-T lights determines that the minimum allowed switch interval is $5 \mathrm{~min}$, which means the lights may be damaged if the switching frequency is higher than twelve times per hour.

The simulation configurations are listed in Table 4. The simulation interval is $5 \mathrm{~min}$, which means that the optimization is executed once every $5 \mathrm{~min}$. The goal of the objective "Fixed Light hours" is configured to be dark between 17:00 on the first day to 07:00 on the second day, so the artificial light must be dark in these hours. "?" means the status of the artificial lights can be either ON or OFF while adhering to this objective. Here, two PAR goals are involved: $10 \mathrm{~mol} / \mathrm{m}^{2} / \mathrm{d}$ and $4 \mathrm{~mol} / \mathrm{m}^{2} / \mathrm{d}$. Simulations are carried out on three different days across the two versions. The following dates have been selected randomly for this purpose: Jan 10, Nov 01 and Dec 20 in 2020. On each date, the simulations according to the two PAR goals are committed on the two versions of DynaLight, respectively. When PAR goal is $10 \mathrm{~mol} / \mathrm{m}^{2} / \mathrm{d}$, it is beyond the maximum collected daily PAR sum, including natural and artificial sources, on the simulation dates. Thus, the cost of the objective "PAR sum balance" always equals one and the cost of the objective "PAR sum balance" is always larger than zero. When PAR goal is $4 \mathrm{~mol} / \mathrm{m}^{2} / \mathrm{d}$, the collected PAR sum of some light-plan can fulfill the goal. That

Table 3 Environmental configuration of short-day compartment

\begin{tabular}{ll}
\hline Item & Configuration \\
\hline Size $\left(\mathrm{m}^{2}\right)$ & 100 \\
Light type & SON-T \\
min allowed switch interval $(\mathrm{min})$ & 5 \\
Rated power $(\mathrm{kW} / \mathrm{h} / \mathrm{bulb})$ & 400 \\
Artificial PAR $(\mu \mathrm{mol} / \mathrm{h} / \mathrm{bulb})$ & 100 \\
PAR conversion rate & $60 \%$ \\
\hline
\end{tabular}


Table 4 Simulation configurations

\begin{tabular}{ll}
\hline Item & Configuration \\
\hline Fixed light hours & {$[0000000$ ??????????0000000] } \\
Simulation interval (min) & 5 \\
PAR goal (mol/m²/day) & 10 or 4 \\
Max generation & 5000 \\
Population size in a generation & 500 \\
Social Welfare metric & Total Utility-Based SW (Utilitarian) \\
Size of light-plan (hours) & 72 \\
\hline
\end{tabular}

is, the costs of objective "PAR sum balance" and "PAR sum balance" can be zero. As a result, the trade-off results vary across the objectives in each vertex, which means that the comparison between the two versions of DynaLight can be more comprehensive. The size of a light-plan solution is 72 , which is the light-plan for the next $72 \mathrm{~h}$ - today and the future 2 days.

\section{Objective importance relation}

In the DynaLight, the importance of objectives is expressed through an absolute priority represented by an integer. The lower the value, the higher the priority. If two objectives have the same priority, they are considered equally important and have a similar nature concerning their objective functions. The priorities of the objectives in the DynaLight are shown in Table 5.

In order to compare the two selection mechanisms, the RIG in DynaLight IND is made consistent with the priority configuration in DynaLight, by using the graph depicted in Fig. 4. In practice, the RIG filters solutions by traversing this graph in a top-down direction and applying the Total Utility-Based SW. In Vertex Root, three objectives are involved, which are "Sufficient PAR sum," "Fixed Light Hours" and "Light Interval." This vertex will filter out solutions that do not adhere to these objectives (are not "best of the Pareto set"). In Vertex 1, trade-off solutions against "PAR sum balance" and "Cheap Light" are selected according to the Total Utility-Based metric and go to Vertex 2. In Vertex 2, the solutions that are with the lowest cost against "Minimum Artificial Light" are selected and transferred to the last vertex. In the last vertex, the light plans that are with the least times of switches are chosen by the evaluation against the objective "Minimum Switches." If there is more than one light plan left after the

Table 5 Priority configuration in DynaLight

\begin{tabular}{lc}
\hline Objective & Priority \\
\hline Sufficient PAR sum & 0 \\
Fixed Light Hours & 0 \\
Light Interval & 0 \\
PAR Sum Balance & 1 \\
Cheap Light & 1 \\
Minimum Artificial Light & 2 \\
Minimum Light Switches & 3 \\
\hline
\end{tabular}




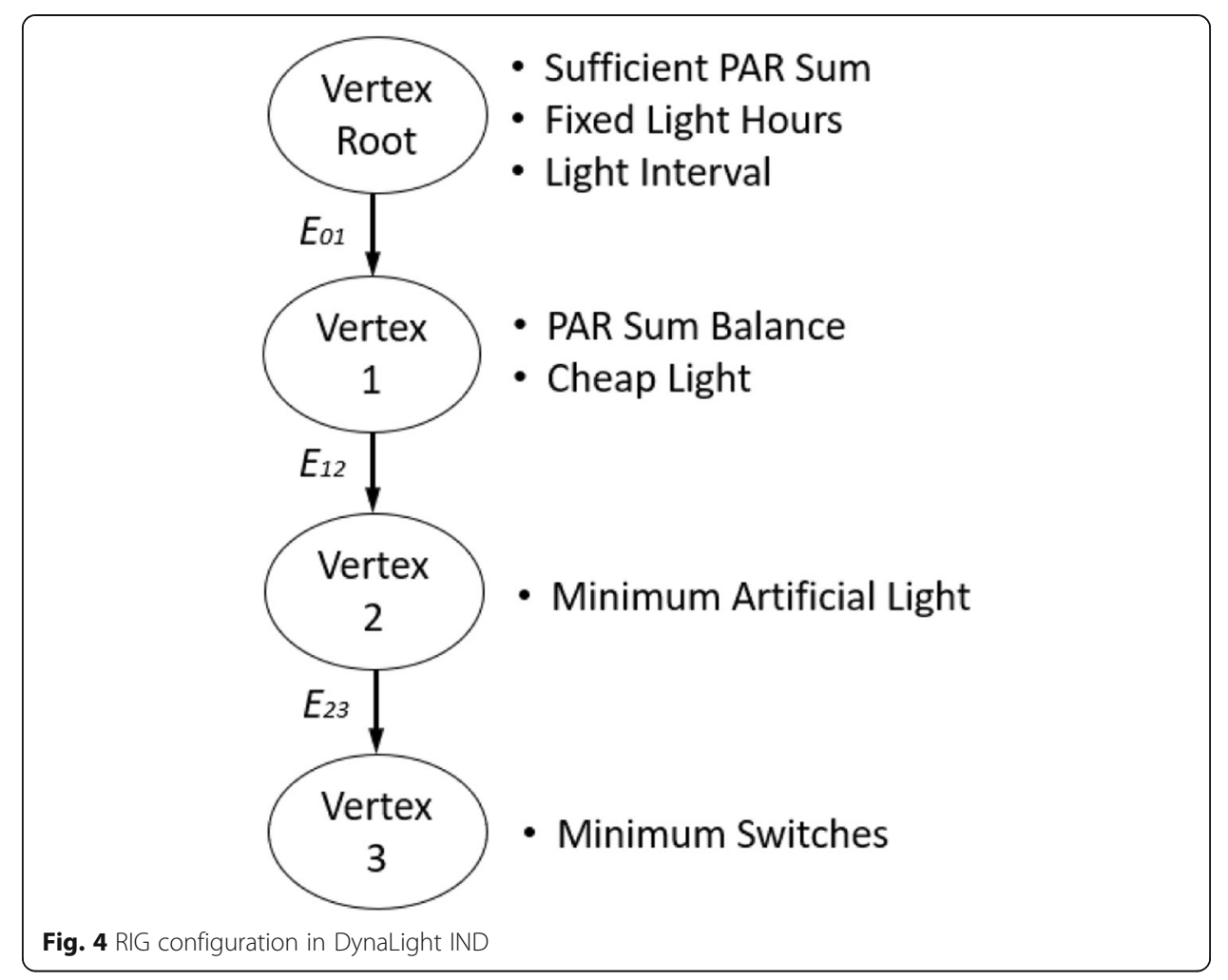

refining of Vertex 3, the final light plan is selected randomly among them, as the remaining solutions are considered equal from the perspectives of the objectives.

In DynaLight IND, after the posteriori normalization, the cost of solutions is converted to a dimensionless quantity that is the ratio against the cost range of a particular objective. Therefore, the normalized cost against the sub-objectives in an individual vertex can directly participate in the SW calculation.

\section{Results}

Simulations are executed three times for each PAR goal on each date, respectively. Table 6 shows the comparison results of the PF sets between DyanLight and DynaLight IND.

In DyanLight IND, due to the average difference of the PF sizes is $0.5 \%$ among the three runs on each date, to compare the coverage of the PF between the two versions

Table 6 Comparison results

\begin{tabular}{|c|c|c|c|c|c|c|c|c|}
\hline \multirow{3}{*}{$\begin{array}{l}\text { PAR goal } \\
\left(\mathrm{mol} / \mathrm{m}^{2} / \mathrm{d}\right)\end{array}$} & \multirow{3}{*}{$\begin{array}{l}\text { Simulation } \\
\text { time }\end{array}$} & \multicolumn{4}{|l|}{ Size of PF } & \multicolumn{3}{|c|}{ Comparison results of PF of DynaLight } \\
\hline & & \multirow{2}{*}{$\begin{array}{l}\text { DynaLight } \\
\text { IND }\end{array}$} & \multicolumn{3}{|c|}{ DynaLight } & \multirow{2}{*}{$\begin{array}{l}\text { Covered by } \\
\text { DynaLight } \\
\text { IND's PF }\end{array}$} & \multirow{2}{*}{$\begin{array}{l}\text { Non- } \\
\text { dominated }\end{array}$} & \multirow[t]{2}{*}{ Dominated } \\
\hline & & & run1 & run2 & $\overline{\text { run3 }}$ & & & \\
\hline \multirow[t]{3}{*}{10} & Jan 10 & 9388 & 152 & & & $96.1 \%$ & 0 & $3.9 \%$ \\
\hline & Nov 01 & 15,025 & 197 & 148 & 148 & $22.8 \%$ & 0 & $77.2 \%$ \\
\hline & Dec 20 & 25,357 & 301 & & & $90.7 \%$ & 0 & $9.3 \%$ \\
\hline \multirow[t]{3}{*}{4} & Jan 10 & 1316 & 2 & 2 & 3 & 0 & 0 & $100 \%$ \\
\hline & Nov 01 & 184 & 2 & 2 & 2 & 0 & 0 & $100 \%$ \\
\hline & Dec 20 & 1207 & 2 & 9 & 3 & 0 & 0 & $100 \%$ \\
\hline
\end{tabular}


of DynaLight, a PF is selected randomly from the three runs of DynaLight IND on each of the three dates.

When the PAR is $10 \mathrm{~mol} / \mathrm{m}^{2} / \mathrm{d}$, DynaLight IND selects the same light plan across the three runs. Regarding DynaLight, the PFs across the three runs are equal on Jan 10 and Dec 20 accordingly, so one PF of the three runs is selected for the comparison with DynaLight IND. But the size of the PFs of run 1 is 197, which is different from the PFs of run 2 and run 3, where the size of the PF is 148 on both occasions. Comparing the three PFs generated by DynaLight on this date, it turns out that the PFs of run 2 and run 3 are all contained in the PF of run1. As a result, the PF of run1 is used for the comparison to give a fair advantage to DynaLight.

When the PAR is $4 \mathrm{~mol} / \mathrm{m}^{2} / d$, the selected light plans and the size of the PF are identical across all runs and dates in DynaLight IND. Therefore, a PF is selected from a random run on each date for comparison with DynaLight. However, in the simulations of DynaLight, the light plans and the solutions in the PFs across the three runs are inconsistent from each other on each simulation date accordingly. As a result, the sizes of PF across the three runs are all listed, and all the three PF participate in the comparison on each date.

The comparison results are listed in Table 6. Comparing each solution in the PF of DynaLight, $s_{\text {old }}^{(i)}(0 \leq i<$ size of $P F)$, with the PF of DynaLight IND, $P F_{I N D}$, and there are three possibilities of the comparison results

- $s_{\text {old }}^{(i)}$ is dominated by $P F_{I N D}$.

- $s_{\text {old }}^{(i)}$ is non-dominated by $P F_{I N D}$, including $s_{\text {old }}^{(i)}$ can dominate some solutions in $P F_{I N D}$ or being only non-dominated without dominating any solutions in $P F_{I N D}$.

- $s_{\text {old }}^{(i)}$.is covered by $P F_{I N D}$.

The light-plan selected by DynaLight and DynaLight IND have been plotted in Figs. 5, 6 and 7 for each of the three dates. Furthermore, the data of the electricity price and the forecast of the PAR intensity have been plotted in the same graphs. The hours that are covered by grey represent the dark hours according to the requirement of "Fixed light hours." The step plots represent the switch status of the artificial lights in light plans. The switch status of a specific hour is ON when the step value is non-zero, and it is OFF when the step value is zero. In situations where there is variance in the light plan selected by DynaLight, each of the light plans has been plotted with a different color dotted line.

In this experiment, the time consumption for the simulation of each generation is $459 \mathrm{~ms}$ on average in DynaLight IND, while it consumes $2587 \mathrm{~ms}$ on average in DynaLight under the consistent simulation configurations.

\section{Discussion}

By analyzing the comparison results in Table 6, when the PAR goal is $10 \mathrm{~mol} / \mathrm{m}^{2} / \mathrm{d}$, the sizes of the PF of DynaLight, $P F_{\text {old }}$, across the three runs are the same on each date except on Nov 01. However, in all cases, their sizes are much smaller than the sizes of the comparable $P F_{I N D}$. When the PAR goal is $4 \mathrm{~mol} / \mathrm{m}^{2} / \mathrm{d}$, the size of $P F_{\text {old }}$ is again much smaller than the size of $P F_{I N D}$ in all cases. Further, the solutions contained in the PFs are inconsistent across the three runs of the simulations of DynaLight. 


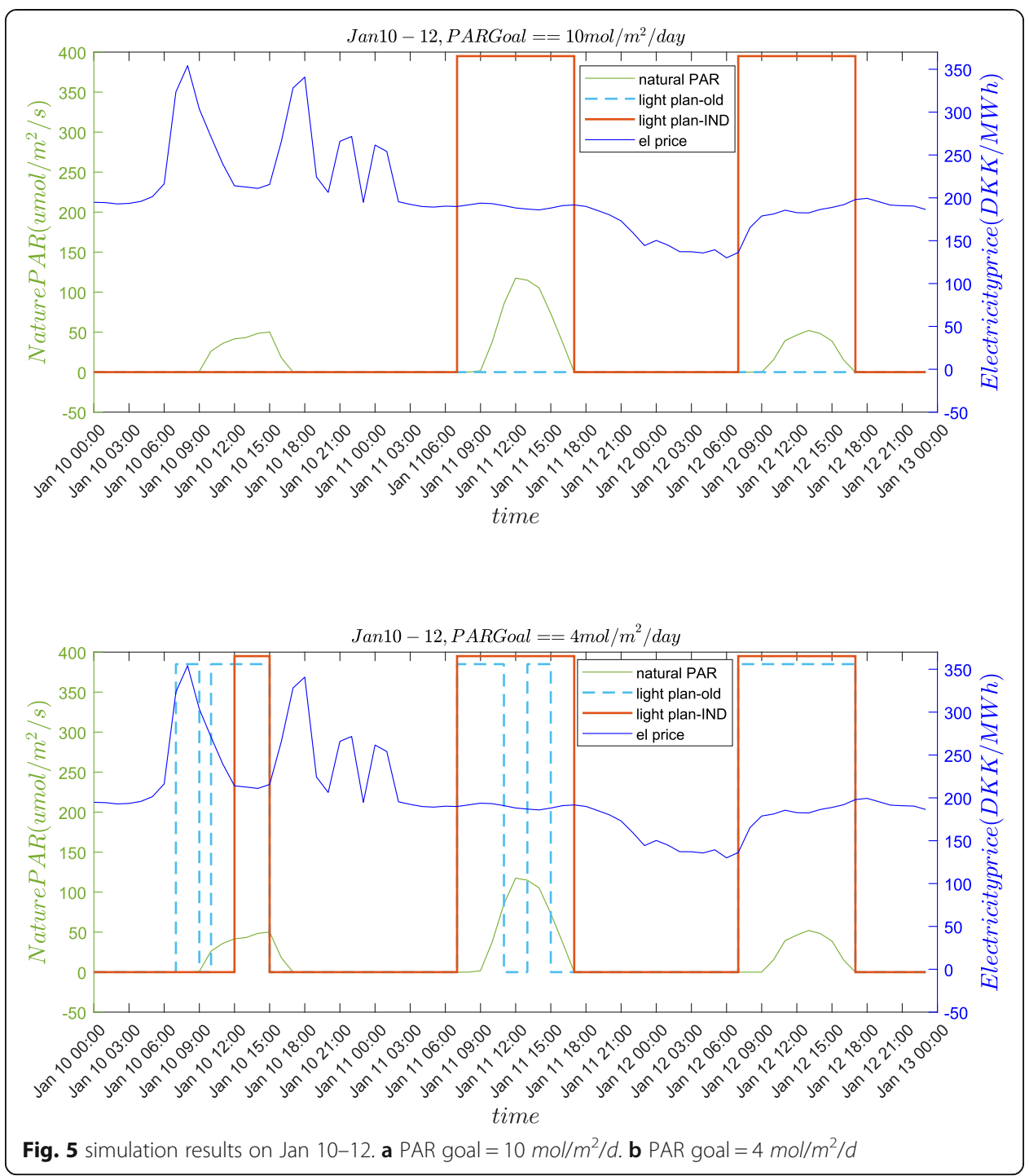

All of the solutions in $P F_{\text {old }}$, namely $s_{\text {old }}^{(i)}$, are dominated by solutions in the corresponding $P F_{I N D}$ under all the PAR goals on each simulation date. When the PAR goal is $10 \mathrm{~mol} / \mathrm{m}^{2} / d$, solutions in $P F_{\text {old }}$ are either contained or dominated by solutions in $P F_{I N D}$ on each date. When the PAR goal is $4 \mathrm{~mol} / \mathrm{m}^{2} / \mathrm{d}$, the solutions in the three runs are all dominated by solutions in the corresponding $P F_{I N D}$ on each date.

In conclusion, the comparison results indicate that the GA implementation strategy of DynaLight causes the PF to be incomplete, which means there some non-dominated solutions are missing. The GA implementation of DynaLight IND denotes an improvement in this direction, as a much broader and more complete PF is generated. Further, the quality of the Pareto set in DynaLight IND is intact or improved according to our findings.

From Figs. 5, 6 and 7, all the light plans of DynaLight and DynaLight IND are acceptable, which means they can fulfill the objectives "Fixed light hours" and "Light interval". When the PAR is $10 \mathrm{~mol} / \mathrm{m}^{2} / d$, on Jan 10 and Dec 20, the light plans are all OFF in the simulations of DynaLight. This is due to the score calculation of solutions is non- 


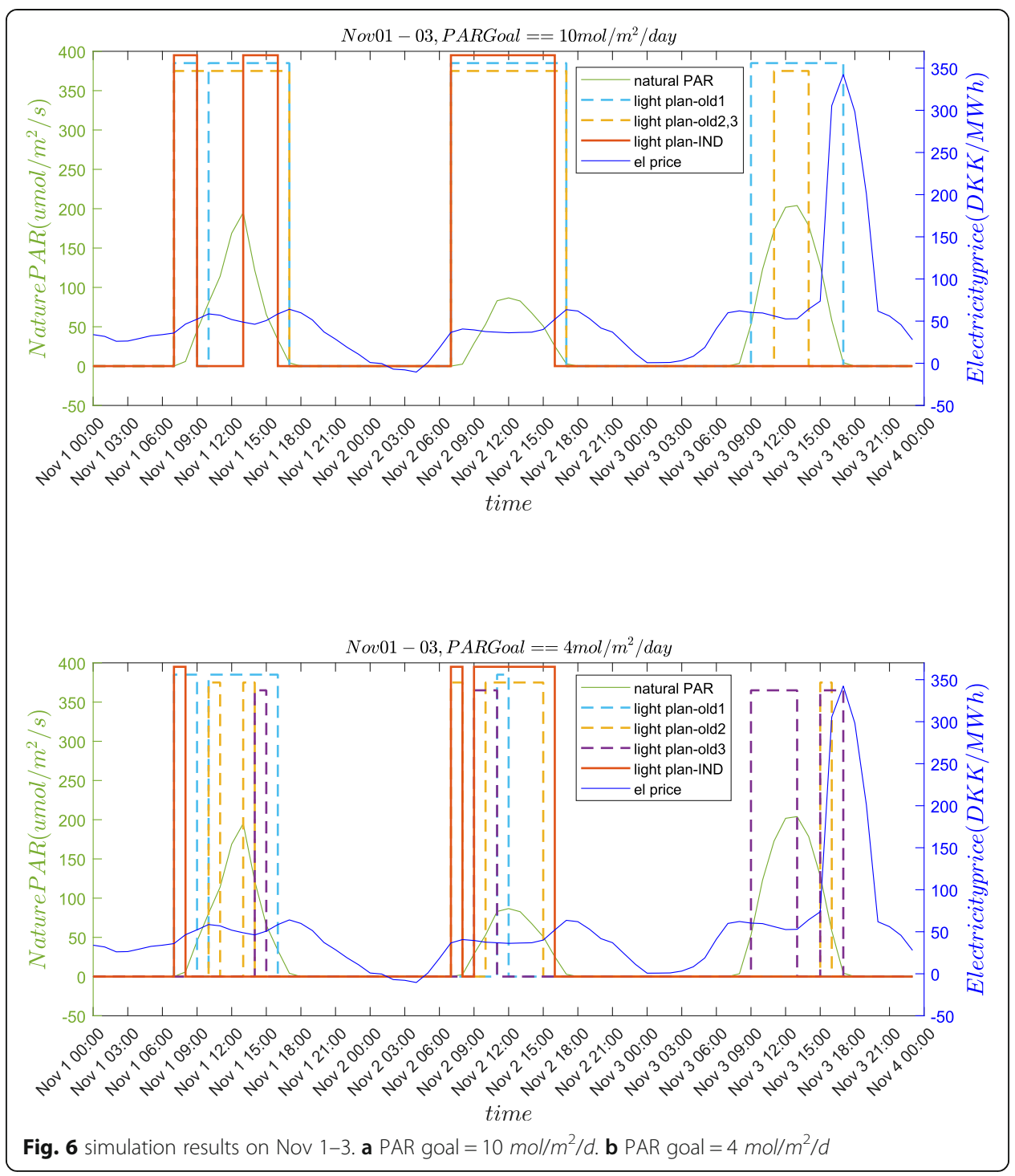

normalized in Vertex 1, which causes the weights of the objectives "PAR sum balance" and "Cheap Light" are different although they are in the same vertex. The ranges of "PAR sum balance" and "Cheap Light" are [0.15, 26.07] and [0.0, 87.20] on Jan 10 and $[0.0,24.34]$ and $[-1.15,58.53]$ on Dec 20 , respectively. Thus, regarding a specific solution, the cost of "Cheap Light" is always much higher than "PAR sum balance" when there is no normalization involved. As a result, the solution selection tends towards fulfilling the objective "Cheap Light" first. In DynaLight IND the normalization that is introduced in the score calculation ensures that the weight of each objective is comparable. Consequently, the filtered light plans are the trade-off results against multiple objectives in a certain vertex.

By analyzing the light plans and the tendency of electricity prices in Figs. 5, 6 and 7, the status ON in the light plans of DynaLight IND can always select the hours that are with relatively lower electricity prices in a period and avoid higher electricity prices on the basis on the requirements of "Fixed light hours". By comparing the average time consumption for each evolution generation in the experiments, the optimization speed 


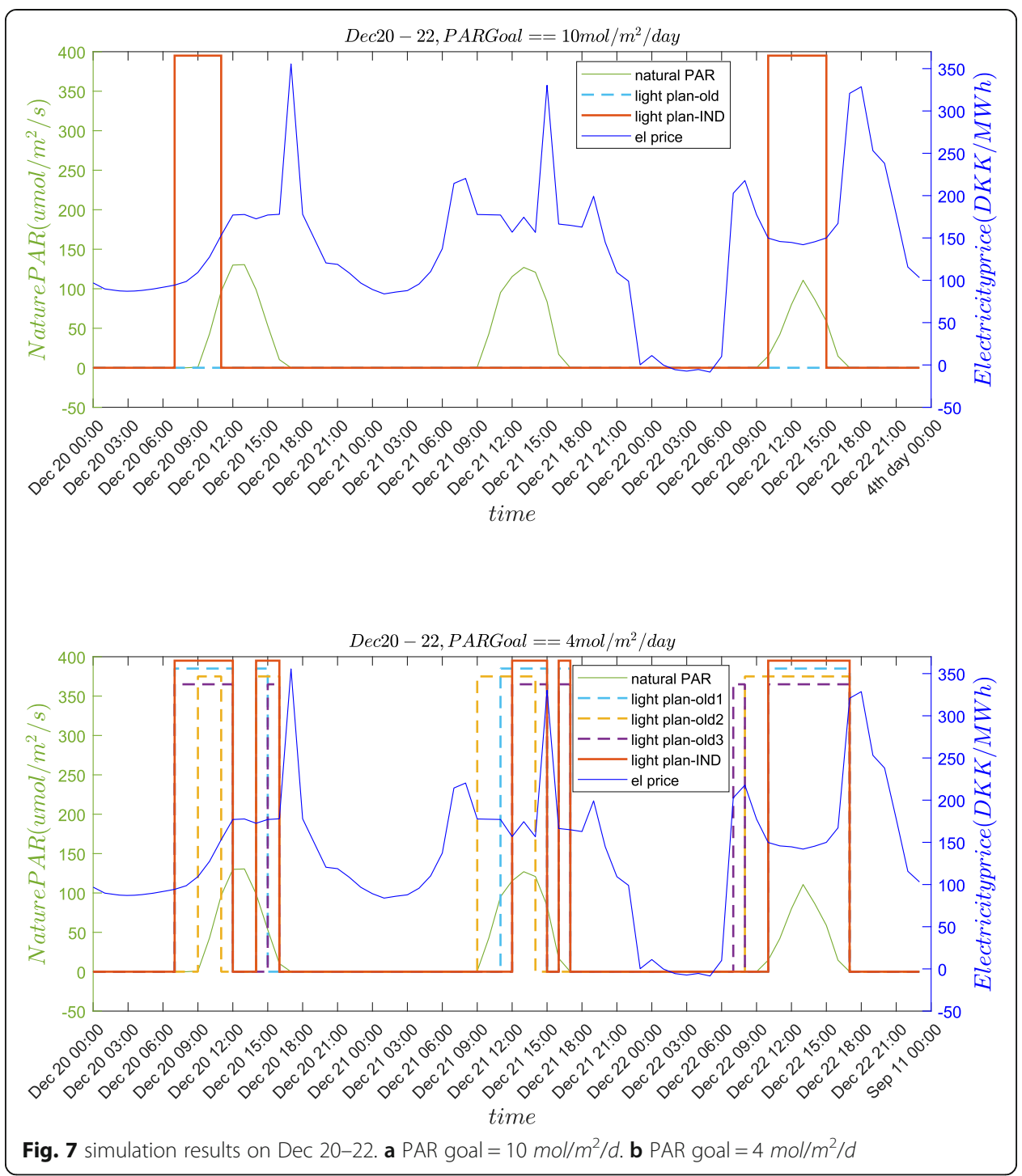

of DynaLight IND is 5.6 times faster than DynaLight on average without compromising the PF coverage under consistent configurations.

\section{Conclusions}

This paper elaborates on the different implementation strategies for the two versions of DynaLight, which is a MOO GA-based platform for optimizing artificial lighting in greenhouses. Firstly the GA-based MOO platform approach taken by DynaLight in, is presented and the reasons for developing DynaLight IND are explained. Secondly, the lighting optimization problem in greenhouse systems is presented and the related objectives are defined. The paper then presents the approach taken in DynaLight IND to overcome the potential problems in DynaLight, including an alteration to the GA evolution strategy and an alternative selection mechanism for finding a solution in a Pareto set.

Simulations have been carried out using the two versions of DynaLight under various scenarios in order to compare the differences of the two evolution strategies. By 
analyzing the simulation results and comparing the results of the two versions, the features of the different evolution strategies are discussed and summarized.

Although some proposed concepts in this paper have been explained and utilized in previous works (Umair et al. 2019; Clausen et al. 2020), such as posterior normalization, social welfare metrics, and relative importance graph, the previous works only focused on a specific implementation point of one of the two versions of the MOO platform DynaLight. In this paper, the entire implementation process of the two versions of DynaLight is elaborated, including the GA evolution process and the final optimal light plan selection. By comparing the pros and cons of the two versions of artificial lighting MOO platforms, this paper contributes to the implementation suggestions regarding improving GA evolution efficiency and increasing computation speed in general areas.

However, there is still some work in this paper that can be improved in future research. For example, when the optimization interval is required to be a short time interval, such as $5 \mathrm{~min}$, time-saving for the optimization process is one of the key points of enhancing the practical properties of DynaLight IND. The solutions to this issue can be considered from two aspects, that are enhancing the calculation efficiency and the calculation ability of hardware. Calculation efficiency can be increased through parallel computing, and calculation ability can be enhanced by using high-performance computers, such as GPU computers. Another limitation is that the properties of DynaLight IND are only verified by simulations, but practical experiments have not been performed. Therefore, some potential issues in practical scenarios, such as the limitations of hardware, cannot be mapped entirely in simulations, even though the features of light bulbs are considered.

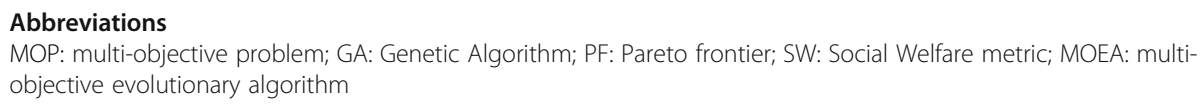

About this supplement

This article has been published as part of Energy Informatics Volume 4, Supplement 2 2021: Proceedings of the Energy Informatics.Academy Conference Asia 2021. The full contents of the supplement are available at https:// energyinformatics.springeropen.com/articles/supplements/volume-4-supplement-2.

\section{Authors' contributions}

YQ performed the simulations and analyzed the results, and then finished the main text of this paper. AN contributed to the problem description and the approach elaboration and also worked on the structure of sections. BNJ contributed to confirming the correction of the concepts and also worked on the structure of the paper and modifying the main text. All authors have read and approved the final manuscript.

\section{Funding}

Danish Energy Technology Development and Demonstration Program (EUDP Project no 64019-0018).

EUDP Project supports private companies and universities to develop and demonstrate new energy technologies.

Support is given in accordance with EU state aid rules.

\section{Availability of data and materials}

The electricity price data, the weather information and the simulation results can be accessed:

https://github.com/yingqu99/DyanLight_simulation_data_EIA2021.git

\section{Declarations}

Ethics approval and consent to participate

Not applicable. 
Consent for publication

Not applicable.

\section{Competing interests}

The authors declare no competing interests.

Published: 24 September 2021

\section{References}

Aaslyng JM, Lund JB, Ehler N, Rosenqvist E (2003) IntelliGrow: a greenhouse component-based climate control system. Environ Model Softw 18(7):657-666. https://doi.org/10.1016/S1364-8152(03)00052-5

Adler M (2012) Well-being and fair distribution: beyond cost-benefit analysis. Oxford Scholarship Online, New York Albadi MH, El-Saadany EF (2008) A summary of demand response in electricity markets. Electr Power Syst Res 78(11):19891996. https://doi.org/10.1016/j.epsr.2008.04.002

Allan RS, Feldman M (2006) Welfare economics and social choice theory. Springer, New York

Anthony Howard D, Ma Z, Mazanti Aaslyng J, Jorgensen BN (2020) Data Architecture for Digital Twin of Commercial Greenhouse Production. In: Proceedings of the 2020 RIVF International Conference on Computing and Communication Technologies (RIVF), Viet Nam, pp 1-7

Chang HF (2000) A liberal theory of social welfare: fairness, utility, and the Pareto principle. Yale Law J 110(2):173-235. https:// doi.org/10.2307/797571

Chevaleyre $Y$ et al (2006) Issues in multiagent resource allocation. Informatica 30:1-37

Clausen A, Umair A, Demazeau Y, Jørgensen BN (2020) Impact of social welfare metrics on energy allocation in multiobjective optimization. Energies 13:1-19

Fonseca CM, Fleming PJ (1998) Multiobjective optimization and multiple constraint handling with evolutionary algorithms part I: a unified formulation. IEEE Trans Syst Man Cybern A Syst Hum 28(1):26-37. https://doi.org/10.1109/3468.650319

Hashem I, Telen D, Nimmegeers P, Logist F, Van Impe J (2017) A novel algorithm for fast representation of a Pareto front with adaptive resolution: application to multi-objective optimization of a chemical reactor. Comput Chem Eng 106:544558. https://doi.org/10.1016/j.compchemeng.2017.06.020

Hemming $S$ et al (2017) Innovations in greenhouse systems - Energy conservation by system design, sensors and decision support systems. Acta Hortic 1170:1-15 Edited: International Society for Horticultural Science

Kjaer KH, Ottosen C-O, Jørgensen BN (2011) Cost-efficient light control for production of two campanula species. Sci Hortic 129(4):825-831. https://doi.org/10.1016/j.scienta.2011.05.003

Kjaer KH, Ottosen C-O, Jørgensen BN (2012) Timing growth and development of Campanula by daily light integral and supplemental light level in a cost-efficient light control system. Sci Hortic 143:189-196. https://doi.org/10.1016/j.scienta.2 012.06 .026

Ma Z, Korsgaard J, Jorgensen BN (2020) Optimization of Greenhouse Production Process: An Investigation of Energy Efficiency Potentials. In: Proceedings of the Proceedings - 2019 6th International Conference on Dependable Systems and Their Applications, DSA 2019, pp 365-370

Markvart J, Kalita S, Nørregaard Jørgensen B, Mazanti Aaslyng J, Ottosen CO (2008) IntelliGrow 2.0 - A greenhouse component-based climate control system. Acta Hortic 801:507-513 PART 1. ^Eds edition

Rytter M, Sørensen J, Jørgensen B, Körner O (2012) Advanced model-based greenhouse climate control using multiobjective optimization. Acta Hortic 957:29-35

Shen Y, Wei R, Xu L (2018) Energy consumption prediction of a greenhouse and optimization of daily average temperature. Energies 11:1-17

Sørensen JC, Kjaer KH, Ottosen CO, Jørgensen BN (2016a) DynaGrow - Multi-objective optimization for energy cost-efficient control of supplemental light in greenhouses. In: Proceedings of the 8th International Joint Conference on Computational Intelligence:1 1, pp 41-48

Umair A, Clausen A, Demazeau Y, Jørgensen BN (2019) Impact of social welfare methods on multi-objective resource allocation in energy systems. In: Proceedings of the 8th International Conference on Smart Cities and Green ICT Systems, pp 179-186

Varian HR (2010) Intermediate Microeconomics: A Modern Approach. W.W.Norton\&Company, New York

Warde D (2015) Greenhouse design and control. Scitus Academics, New York

\section{Publisher's Note}

Springer Nature remains neutral with regard to jurisdictional claims in published maps and institutional affiliations. 